\title{
A Problem Solving Environment for Image-Based Computational Hemodynamics
}

\author{
Lilit Abrahamyan ${ }^{1, \star}$, Jorrit A. Schaap ${ }^{2}$, Alfons G. Hoekstra ${ }^{1}$, \\ Denis Shamonin ${ }^{1}$, Frieke M.A. Box ${ }^{2}$, Rob J. van der Geest ${ }^{2}$, \\ Johan H.C. Reiber ${ }^{2}$, and Peter M.A. Sloot ${ }^{1}$ \\ 1 Section Computational Science, Laboratory for Computing, \\ System Architecture and Programming, Faculty of Science, \\ University of Amsterdam Kruislaan 403, \\ 1098 SJ Amsterdam, The Netherlands \\ \{labraham, alfons, dshamoni, peter\}@science.uva.nl \\ http://www.science.uva.nl/research/scs/ \\ 2 Division of Image Processing, Department of Radiology, \\ Leiden University Medical Center Albinusdreef 2, 2333 ZA Leiden, \\ PO Box 9600, 2300 RC Leiden, The Netherlands \\ \{J.A.Schaap, J.H.C.Reiber, R.J.van_der_Geest, F.M.A.Box $\}$ @lumc.nl \\ http://www.Ikeb.nl
}

\begin{abstract}
We introduce a complete problem solving environment designed for pulsatile flows in 3D complex geometries, especially arteries. Three-dimensional images from arteries, obtained from e.g. Magnetic Resonance Imaging, are segmented to obtain a geometrical description of the arteries of interest. This segmented artery is prepared for blood flow simulations in a 3D editing tool, allowing to define in- and outlets, to filter and crop part of the artery, to add certain structures ( e.g. a by-pass, or stents ), and to generate computational meshes as input to the blood flow simulators. Using dedicated fluid flow solvers the time dependent blood flow in the artery during one systole is computed. The resulting flow, pressure and shear stress fields are then analyzed using a number of visualization techniques. The whole environment can be operated from a desktop virtual reality system, and is embedded in a Grid computing environment.
\end{abstract}

Keywords: Problem Solving Environment, Computational Hemodynamics, blood flow modeling.

\section{Introduction}

"A problem solving environment (PSE) is a computer system that provides all the computational facilities necessary to solve a target class of problems" [1,2]. The target class of problems that we chose in our study is associated with cardiovascular diseases, a predominant cause of death [3, 4. In particular our attention

\footnotetext{
* Corresponding author.
} 
is concentrated on vascular disorders caused by atherosclerosis. The goal of our PSE, which we call HemoSolve, is to provide a fully integrated environment for simulation of blood flow in patient specific arteries.

Because of the complex structure of the human vascular system it is not always obvious for surgeons how to solve the problem of bypass and/or stent placement on the deformed part, or to decide on specific treatment alternatives. Having a completely integrated computational hemodynamics environment like HemoSolve can serve as a pre-operational planing tool for surgeons, but also as a useful experimental system for medical students to enlarge their practical skills [5,6]. It also serves as a environment for biomedical engineers that study e.g. new stent designs.

Moreover, our HemoSolve is merged with Grid technology, thus offering a unified access to different and distant computational and instrumental resources [6]. This is one of the desirable abilities of PSEs in general [2].

We first describe our system for image-based computational hemodynamics, then we provide examples of using it in the case of the abdominal aorta bifurcation and an abdominal aorta aneurysm, and finally the last section represents discussions and conclusions.

\section{The Problem Solving Environment - HemoSolve}

In ref. 5] Steinman argues that a need exists for robust and user-friendly techniques that can help an operator turn a set of medical images into computational fluid dynamics (CFD) input file in a matter of minutes. HemoSolve not only has this ability but also is a tool which allows to simulate pulsatile (systolic) flows in arteries.

The whole system consists of the following components (See Fig,1):

1. Medical data segmentation to obtain arteries of interest;

2. 3D editing and mesh generation, to prepare for the flow simulation;

3. Flow simulation, computing of blood flow during systole;

4. Analyses of the flow, pressure, and stress fields.

\subsection{Medical Data Segmentation}

The goal of the segmentation process is to automatically find the lumen border between the blood and non-blood, i.e. the vessel wall, thrombus or calcified plaque. The algorithm consists of three stages: In the first stage a wave front propagation algorithm is used to find an approximation of the centerline of the vessel. In the second stage the volumetric data is resampled into a stack of $2 \mathrm{D}$ slices orthogonal to the centerline. Then in each slice a contour delineating the lumen border is detected. Finally in the third stage, the stack of $2 \mathrm{D}$ contours is combined to form a 3D surface model, which will serve as input for the $3 \mathrm{D}$ editing tool. 


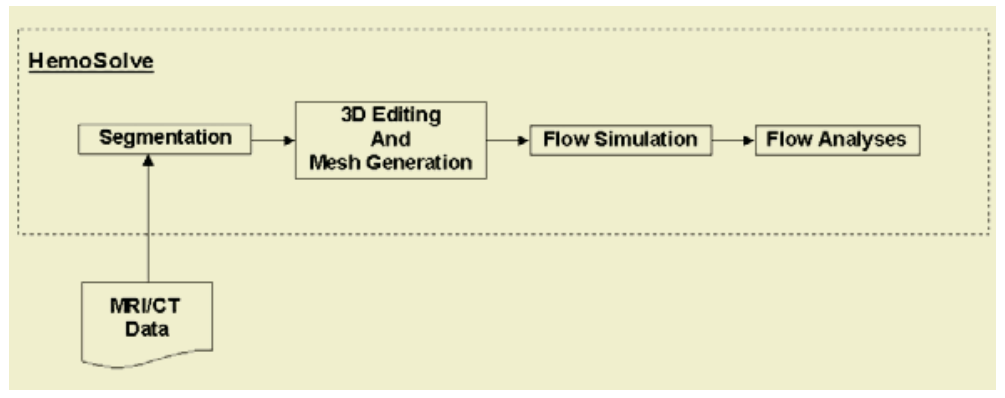

Fig. 1. Functional design of HemoSolve

3D Centerline Extraction: In order to get a first estimate of the centerline of the vessel we use the WaveProp method as described in [7] and [8. WaveProp is based on the Fast Marching Level Set algorithm introduced by Sethian [9]. The principle is that an image is treated as an inhomogeneous medium through which a wave can propagate. The speed of the wave front is determined by a speed function which maps intensity values to speed values. The propagating wavefront creates a monotonous ascending function with its single minimum at the startpoint; with a steepest decent the shortest path from end point to startpoint is found.

In the 3D computer tomography angiography (CTA) datasets the user indicates with two points the beginning (proximal point) and end (distal point) of the vessel segment of interest. Then, a wave front is initiated at the proximal point and propagated through the vessel until it reaches the distal point marking all visited voxels as lumen (blood pool). This gives us a binary volume of the solid lumen. A distance image [10,11] is then calculated from the binary lumen volume containing for each voxel its distance to the background. Consequently, there will be a 3D ridge of high values in the distance image, which coincides with the exact path of the centerline of the solid blood pool. This ridge can be tracked as described in [8] using wave front Propagation and Backtracking, resulting in the centerline ( Fig. 2).

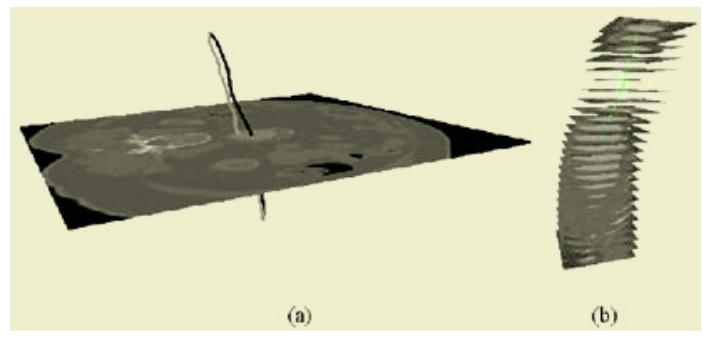

Fig. 2. (a) - is the pathline and the centerline crossing an axial slice, (b) - is a stack of slices resampled along the centerline 
Per Slice Contour Detection: Perpendicular to the detected centerline 2D slices are extracted from the original CTA dataset ( Fig. 2). In each of these transversal slices, a contour delineating the lumen is detected using the WaveProp algorithm similar as it was used to detect the centerline, but now with different speed functions and cost images. Contours delineating objects in an image usually follow the edge of that object. With the proper speed function, a wave propagating through an edge image will flow fast along the object edges and stall in solid parts of the object and in the background. The backtrack method can be used to obtain a contour from one point on the edge to another point on the edge, following that particular edge.

3D Surface Model: The stack of 2D transversal contours can be used to obtain per-slice information, such as diameter, circumference and area of the 2D contour. For the flow simulations however, a 3D surface model is needed. Therefore, the stack of $2 \mathrm{D}$ contours is converted into a $3 \mathrm{D}$ surface model by connecting each of the contour points of one slice to the closest contour point in the next slice ( Fig. 4 (a)).

\subsection{D Editor and Mesh Generator}

$3 \mathrm{D}$ editing is the second component after the segmentation. The 3D stereoscopic image can easily be maintained in this user-friendly editing tool. Here surgeons and students can execute their experimental visualization studies on realistic arterial geometries. They can crop parts of the artery, where important factors in the study of hemodynamics exist, with the help of a clipping instrument. They can add inlet and outlet layers on the end-points of the arterial geometry and can enhance it with structures like bypasses or stents. Also this component allows them to define the geometrical features ( e.g. width, length, placement positions ) of these structures. Thus, the 3D editing tool allows surgeons and students to mimic the real surgical processes.

The final stage of this component is mesh generation. The prepared arterial geometry, including aneurysms, bifurcations, bypasses and stents, is converted into a computational mesh in several minutes. The mesh could be coarse or fine depending on the wish of user.

The mesh is then ready to be used in flow simulators.

\subsection{Hemodynamic Solvers}

Two different computational hemodynamic solvers can be used in HemoSolve:

1. Lattice Boltzmann method (LBM)

2. Finite element method (FEM)

In both solvers the flow is time-dependent and after simulation the pressure, velocity, and shear stress fields during one full systolic period are produced and can be visualized. Both solver receive the input geometry mesh from the 3D editing tool. 
LBM is a mesoscopic method based on a discretized Boltzmann equation with simplified collision operator 12. Here the flow is considered Newtonian. In the solver bounce back on links is used as boundary condition on walls and pressure difference boundary condition is applied on inlet/outlets. We have shown that LBM is capable of solving hemodynamic flows in the range of Reynolds and Womersley numbers of interest [13].

To run the simulator, except the input data file from $3 \mathrm{D}$ editing tool, one should define several patient-specific free parameters like Reynolds number.

FEM is a general discretization tool for partial differential equations. For blood flow model the incompressible Navier-Stokes equations are used. Input parameters are the velocity profiles of the in- and outflow of the bifurcation and a model which calculates the non-Newtonian properties of the blood in a patient specific manner [14. The finite element package that was used in this study is called SEPRAN [15].

\subsection{Flow Analyses}

In order to analyze the blood flow in arteries its velocity, pressure and shear stress profiles need to be examined. Several methods exist for it and among them visualization of the flow is one of the advanced methods that helps to understand the meaning and behavior of flow better. Also visualization techniques are different and can show different features of flow. One of the visualization techniques we use in HemoSolve is based on simulated pathline visualization [16].

\section{$3 \quad$ Examples}

As an application example of using HemoSolve we present two case studies complex geometries representing parts of the human vascular system:

1. Aneurysm on upper part of abdominal aorta;

2. Whole abdominal aorta.

\subsection{Aneurysm}

We consider case of an aneurysm ( ballooning out of the artery ) in the upper part of the aorta. First the medical data of the upper part of patient's abdominal aorta with aneurysm is segmented by applying the segmentation algorithm ( Fig 3 (a,b) ). Then the segmented part which includes the aneurysm, is transfered into $3 \mathrm{D}$ editing tool where the user crops the structurally interesting part (Fig 3 (c) ) and defines inlet and outlet layers ( Fig $3(\mathrm{~d})$ ). This placement is easy to control, that is to change the plane of position with simply moving the normal vector in the middle of the layer or to change the size just by movement of corner points of the rectangle. In this example there is one inlet at the top 


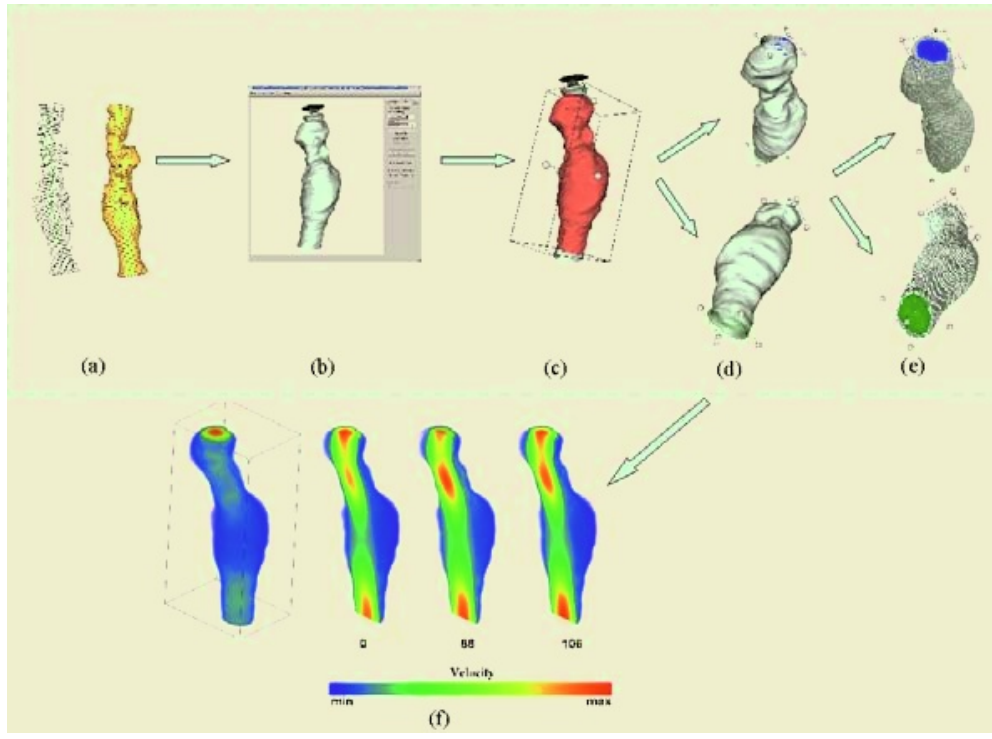

Fig. 3. Aneurysm: main stages of HemoSolve. First segmentation of the raw medical data (a). Then the segmented data (b) is first cropped (c) and inlet/outlet layers are added (d) and the mesh is generated (e). Simulation results of created mesh are presented (f)

and one outlet at the bottom layer. Finally the mesh is generated depending on constructed geometry ( Fig 3 (e) ). The mesh is then used as an input data for CFD solver. The presented flow is the velocity profile of blood flow simulated by LBM method. The Reynolds number applied to this blood flow is 500. The size of generated mesh is $146 \times 73 \times 55$ lattice points and the simulation time is about 20 minutes on 16 processors. As a result three frames during the cardiac systole are captured ( Fig 3 (f) ).

\subsection{Abdominal Aorta}

Next we consider the full lower abdominal aorta down to the bifurcation. Again the medical data is segmented by applying the segmentation algorithm ( Fig 4 (a) ). Then the same steps as in the first example are applied ( Fig 4 (b)), except the outlet layers here are six and are laing in different planes ( Fig 4 (c) ) and the sizes of generated mesh are bigger ( Fig. 4 (d) ). The ready mesh then used as an input data for CFD solver. The presented flow is the velocity profile of blood flow simulated by LBM method with the same Reynolds number 500 . The size of generated mesh is $355 \times 116 \times 64$ lattice point and the simulation time is 85 minutes on 16 processors. As a result three frames during cardiac systole are captured ( Fig 4 (e) ). 


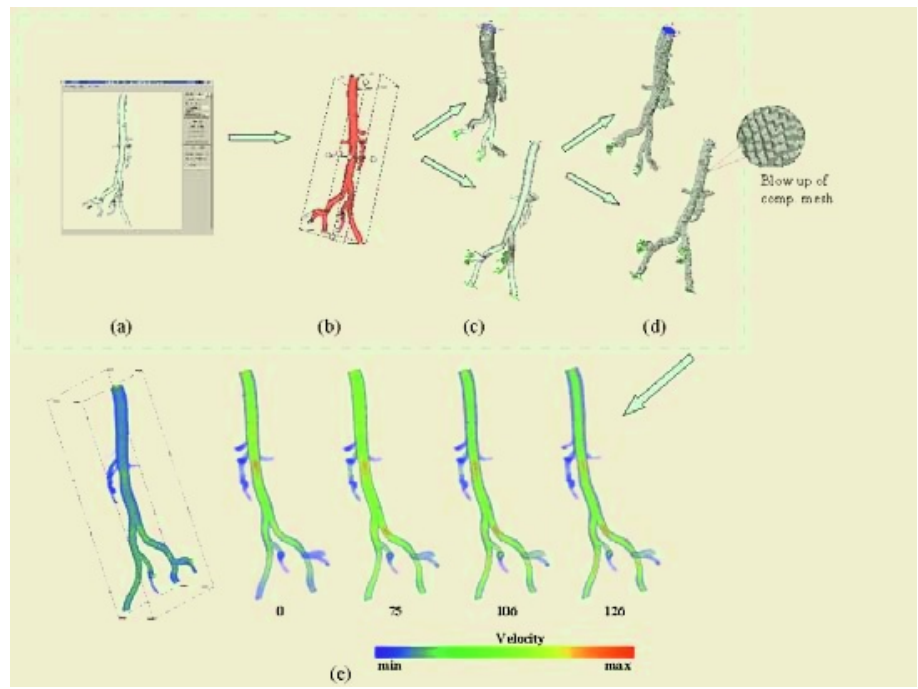

Fig. 4. Abdominal aorta: main stages of HemoSolve. Segmented data (a) is first cropped (b) and inlet/outlet layers are added (c) and the mesh is generated (d). Simulation results of created mesh are presented (e)

\section{Discussions and Conclusions}

The field of image based hemodynamics needs integrated PSEs, especially to enhance the preparation of computational meshes, and to allow non-specialists to enlarge their practical skills. HemoSolve consists of several stages that make it a complete system. Thus biomedical engineers,surgeons or novice surgeons can take a raw medical data from patients vascular system and after several simple steps within a quite short period can get completed flow fields ( velocity, pressure, shear stress) and can analyze them with different visualization tools. One of this tools is personal space station (PSS) which realize $3 \mathrm{D}$ visualization and interaction [17. Moreover with the help of 3D editing tool potential users can add bypasses or stents to vessel and examine the blood flow profile in them. The biomechanists of University of Amsterdam and Leiden University Medical Center have already used Hemosolve in their scientific research.

In order to estimate the efficiency of HemoSolve we compare its main features with the requirements of users from PSEs in general. Those characteristics are [1, 2] :

1. Simple human-computer interaction - It is enhanced by graphical user interface which is easy accessible even for inexperienced users [17].

2. Complete and accurate numerical models - Numerical models used in this PSE are LBM [13] and FEM [14,15] which are complete and quite accurate for simulation of blood flow in human vascular system.

3. Parallel and distributed computing environment - The solvers in PSE are fully parallelized [18] . 
4. Geographically distributed data - This image-based PSE is completely integrated into a Grid environment, which gives huge abilities not only to distribute the data but also to do simulations and diagnoses by grid computing [6] .

5. Usefulness for university student - The potential users of this PSE are considered novice surgeons who can first practice their knowledge by doing an operation on PSE and afterward apply their experience on patients.

Thus, we conclude that HemoSolve is a well defined, easy applicable environment for image-based hemodynamics research of time-dependent blood flow in the human vascular system.

\section{Acknowledgements}

The work was funded by the Dutch National Science Foundation, NWO, Token 2000 Distributed Interactive Medical Exploratory for 3D Medical Images (DIME) project ( 634.000.024).

\section{References}

1. E.Gallopoulos, E.N. Houstis and J.R. Rice, IEEE Comp. Sci. Eng. 1 2, 11 (1994);

2. E.N. Houstis and J.R. Rice, Math. and Comp. in Sim. 54, 243 (2000);

3. Ch.A. Taylor and M.T. Draney, Annu. Rev. Fluid. Mech. 36, 197 (2004);

4. World Health Organization, 2002, The World Health Report 2002, http://www.who.int/whr/en/ ;

5. D.A. Steinman, Annals of Biom. Eng. 30, 483 (2002);

6. A. Tirado-Ramos, P.M.A. Sloot, A.G. Hoekstra and M. Bubak, Parallel Computing 30, 1037 (2004);

7. J.P. Janssen, G. Koning, P.J.H. de Koning, J.C. Tuinenburg and J.H.C. Reiber, Int J Cardiovasc Imaging 18317 (2002);

8. P.J.H. de Koning, J.A. Schaap, J.P. Janssen, R.J. van der Geest and J.H.C. Reiber, Magnetic Resonance in Medicine 501189 (2003);

9. J.A. Sethian, Proc Nat Acad Sci USA 931591 (1996);

10. T. Saito and J.I. Toriwaki, Pattern Recognition 271551 (1994);

11. O. Cuisenaire, PhD thesis, Universit_e catholique de Louvain (October, 1999);

12. S. Succi, New York:Oxford (2001);

13. A.M. Artoli, A.G. Hoekstra and P.M.A. Sloot, Int. J. of Mod. Phys. B, 1795 (2003);

14. F.M.A. Box, M.C.M. Rutten, M. A. van Buchem, J. Doornbos, R.J. van der Geest, P.J.H. de Koning, J. Schaap, F.N. van de Vosse and J.H.C. Reiber Computational Science - ICCS 2002, 255 (April, 2002);

15. G. Segal, Ingenieursbureau SEPRA, Park Nabij 3, Leidschendam, the Netherlands

16. D.A. Steinman, Jour. of Biomech. 33 5, 623 (2000);

17. E.V. Zudilova and P.M.A. Sloot Springer-Verlag Berlin Heildelberg 3345184 (2005).

18. D. Kandhai, A.G. Hoekstra, M. Kataja, J. Timonen and P.M.A. Sloot, Comp. Phys. Commun., 11114 (1998); 